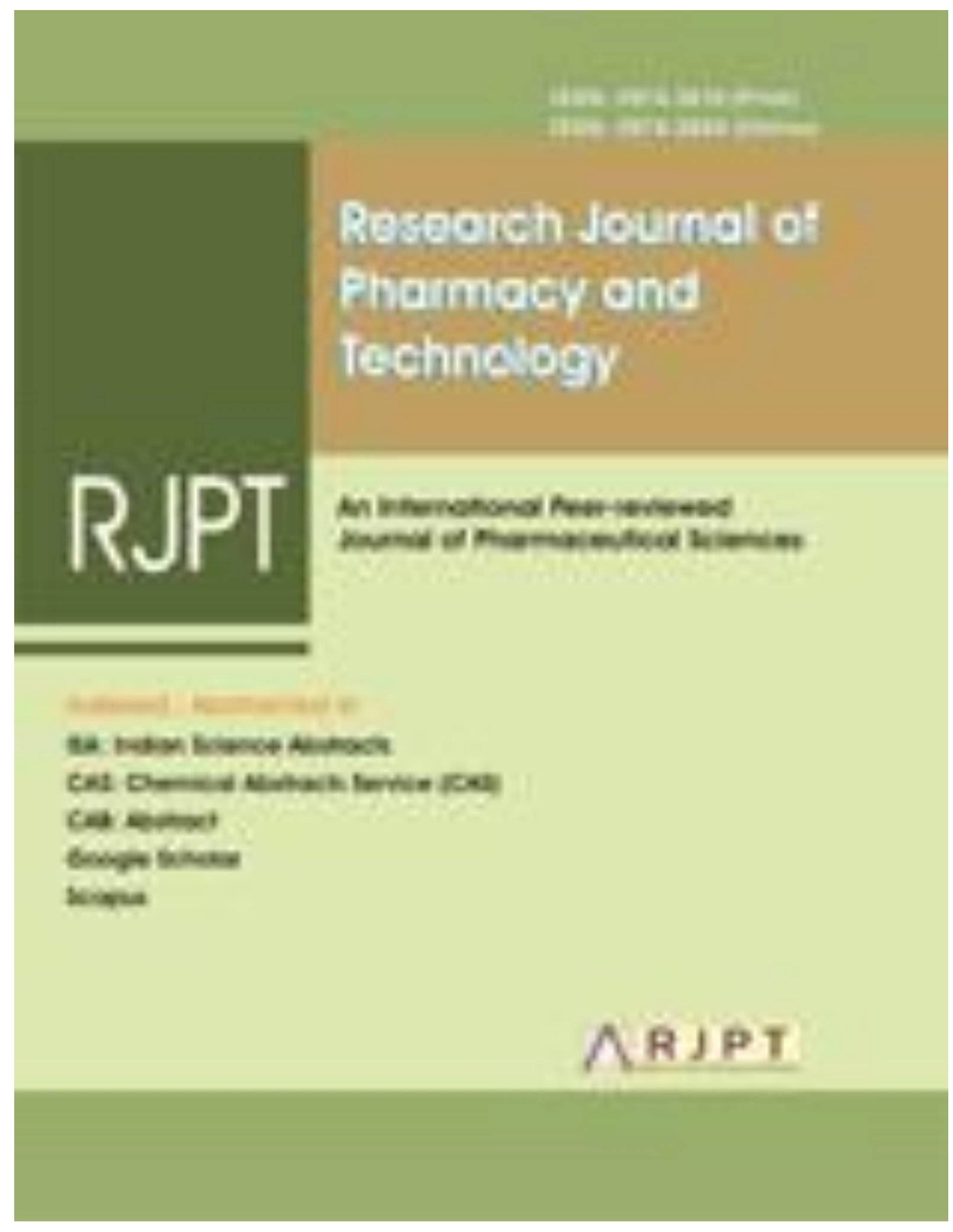




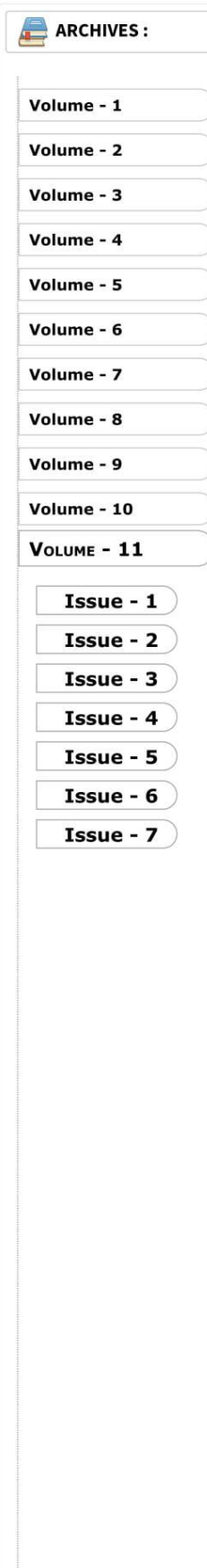

http://rjptonline.org/Archives.aspx
Volume - $11 \mid$ Issue - 5

Online since Wednesday, May 30, 2018

Accessed 5779 times.

Download Cover Image

ORIGINAL ARTICLES

Design and Characterization of Quick Dissolving Film for Simultaneous Delivery of Montelukast Sodium and Levocetirizine Dihydrochloride (AbstractView.aspx?PID=2018-11-5-1)

Savita Kengeri, Nagesh C, Suma Naduvinamani, Sunil L Attimarad, Devika Jorapur, Chandrasekhara S, Sudhir N Sambrekar

DOI: 10.5958/0974-360X.2018.00317.7

[Abstract] [PDF Paper] [HTML Paper] (HTMLPaper.aspx? Journal=Research Journal of Pharmacy and

Technology;PID=2018-11-5-1) [Cite] iflewed: 0 (pdf), 216 (html) Private Access

Bioactive Potential of Fermented Beetroot juice with Pediococcus pentosaseus (VITMS07) (AbstractView.aspx? PID=2018-11-5-10)

V. Mohanasrinivasan, Tiyash Dasgupta, Anatara Homeroy, Nivetha. A

DOI: 10.5958/0974-360X.2018.00326.8

[Abstract] [PDF Paper] [HTML Paper] (HTMLPaper.aspx? Journal=Research Journal of Pharmacy and

Technology;PID=2018-11-5-10) [Cite]

Assessing the Effectiveness of Immunization Reminder System among nursing Mothers of South India

(AbstractView.aspx?PID=2018-11-5-11)

Ketaki Shindel, Usha Rani, Naveen Kumar $P$

DOI: $10.5958 / 0974-360 X 2018.00327 X$

[Abstract] [PDF Paper] [HTML Paper] (HTMLPaper.aspx? Journal=Research Journal of Pharmacy and

Technology:PID=2018-11-5-11) [Cite]

Fill Viewed: 0 (pdf), 91 (html) in Private Access

Formulation and Evaluation of Self-Poring Osmotic Tablet of Diltiazem HCl for the Treatment of Hypertension (AbstractView.aspx?PID=2018-11-5-12)

Hemlata Thapa, Amit Alexander, Tripti Banjare, Palak Agrawal Akansha Bhandarkar, Aditi Bhatt, Swapnil Gupta, Hemlata Sahu, Shradha Devi Divedi, Pankaj Sahu, Siddharth Kumar Sahu, Pooja Yadav, Kailash Sahu, Deeksha Dewang Deepika, Mukesh Sharma, D. K. Tripathi Ajazuddin DOI: $10.5958 / 0974-360 X .2018 .00328 .1$

[Abstract] [PDF Paper] [HTML Paper] (HTMLPaper.aspx? Journal=Research Journal of Pharmacy and Technology;PID=2018-11-5-12) [Cite] Piewed: 0 (pdf), 108 (html) Private Access

The Strategy to Achieve Universal Health Coverage Membership in Indonesia (AbstractView.aspx?PID=201811-5-13)

Arlina Dewi, Ali Ghufron Mukti

Dol: $10.5958 / 0974-360 \times 2018.00329 .3$

[Abstract] [PDF Paper] [HTML Paper] (HTMLPaper.aspx? Journal=Research Journal of Pharmacy and

Technology; $\mathrm{PID}=2018-11-5-13$ ) [Cite]

Screening and characterization of $\beta$-Galactosidase producing Lactobacillus sp. isolated from dairy samples (AbstractView.aspx?PID=2018-11-5-14)

Nivetha. A, Mohanasrinivasan

DOI: $10.5958 / 0974-360 X .2018 .00330 X$

[Abstract] [PDF Paper] [HTML Paper] (HTMLPaper.aspx? Journal=Research Journal of Pharmacy and

Technology:PID=2018-11-5-14) [Citel

Fill Viewed: 0 (pdf), 90 (html) in Private Access

To Study the Awareness on Hygiene Issues-A Survey (AbstractView.aspx?PID=2018-11-5-15) 
8/0974-360X.2018.00331.1

[Abstract] [PDF Paper] [HTML Paper] (HTMLPaper.aspx? Journal=Research Journal of Pharmacy and

Technology;PID=2018-11-5-15) [Cite]

Colorimetric Analysis of Raltegravir in Pharmaceutical Formulation and Human Biological Fluids through Oxidative Coupling Reaction using 3-Methyl-2-Benzothiazolinone Hydrazone Reagent (AbstractView.aspx? PID=2018-11-5-16)

Uttam Prasad Panigrahy, Siva Prasad Panda, Muvvala Sudhakar

DOl: 105958/0974-360X 201800332.3

[Abstract] [PDF Paper] [HTML Paper] (HTMLPaper.aspx?Journal=Research Journal of Pharmacy and

Technology:PID=2018-11-5-16) [Cite]

Fill Viewed: 0 (pdf), 83 (html) A. Private Access

Feasibility of Squash Cytology as a Diagnostic Technique in oral Squamous Cell Carcinoma -A Pilot Study (AbstractView.aspx?PID=2018-11-5-17)

Aiman Zafar, Pratibha Ramani, Anuja N,

[Abstract] [PDF Paper] [HTML Paper] (HTMLPaper.aspx? Journal=Research Journal of Pharmacy and

Technology:PID=2018-11-5-17)

pll viewed: 0 (pdf), 100 (html) APrivate Access

A Comprehensive Study through Training to Prevent Dry Eye Syndrome among Mobile Phone Addicts (AbstractView.aspx?PID=2018-11-5-18)

Seong-Ran Lee

DOI: $10.5958 / 0974-360 \times .2018 .00334 .7$

[Abstract] [PDF Paper] [HTML Paper] (HTMLPaper.aspx? Journal=Research Journal of Pharmacy and Technology; $P I D=2018-11-5-18$ ) [Cite]

Bioprospecting of Clot Buster Enzyme Producing Staphylococcus sp from Bovine Milk Sample (AbstractView.aspx?PID=2018-11-5-19)

V. Mohanasrinivasan, Gurav Rawat, A

[Abstract] [PDF Paper] [HTML Paper] (HTMLPaper.aspx? Journal=Research Journal of Pharmacy and Technology;PID=2018-11-5-19) [Cite]

Influence of Different Doses of Vermicompost and NPK on Growth of Herb Ocimum tenuiflorum var. CIM-Ayu (AbstractView.aspx?PID=2018-11-5-2)

A Ranganadha Reddy, K. S. Ravi Teja,

[Abstract] [PDF Paper] [HTML Paper] (HTMLPaper.aspx? Journal=Research Journal of Pharmacy and Technology;PID=2018-11-5-2) [Cite]

Botanicals to Control Post-Harvest Decay of Colocasia (Colocasia esculenta L.) Tubers in Odisha, India (AbstractView.aspx?PID=2018-11-5-20)

Akhtari Khatoon, Ashirbad Mohapatra, Kunja Bihari Satapathy

DOl: $10.5958 / 0974-360 \times 201800335.9$

[Abstract] [PDF Paper] [HTML Paper] (HTMLPaper.aspx? Journal=Research Journal of Pharmacy and

Technology:PID=2018-11-5-20) [Cite]

Fill Viewed: 0 (pdf), 55 (html) At Private Access

Antioxidant Defense and Disease activity in Rheumatoid Arthritis (AbstractView.aspx?PID=2018-11-5-21)

B. Premkum

DOI: 10.5958/0974-360X.2018.00336.

[Abstract] [PDF Paper] [HTML Paper] (HTMLPaper.aspx?Journal=Research Journal of Pharmacy and

Use of Syzgium aromaticum (Clove) in combination with standard antibiotics: Application in antimicrobial chemotherapy (AbstractView.aspx?PID=2018-11-5-22)

Himanshu Singh, Rajneet Kaur, Shweta Sing

DOI: $10.5958 / 0974-360 X .2018 .00337 .2$

[Abstract] [PDF Paper] [HTML Paper] (HTMLPaper.aspx? Journal=Research Journal of Pharmacy and

Technology;PID=

Development and Application of an Analytical Method for Salivary Inorganic Anion Quantification for the Diagnosis of Systemic and Oral Diseases (AbstractView.aspx?PID=2018-11-5-23)

Jung-Eun Park, Jong-Hwa Jang

DOl: $10.5958 / 0974-360 \times .2018 .00338 .4$ 
[Abstract] [PDF Paper] [HTML Paper] (HTMLPaper.aspx? Journal=Research Journal of Pharmacy and Technology;PID=2018-11-5-23) [Cite] All Viewed: 0 (pdf), 89 (html) Private Access

Awareness of Diabetes Mellitus among General Population (AbstractView.aspx?PID=2018-11-5-24)

S. Preethikaa, M. P. Brundha

DOI: 10.5958/0974-360X2018.00339.

[Abstract] [PDF Paper] [HTML Paper] (HTMLPaper.aspx?Journal=Research Journal of Pharmacy and

Technology:PID=2018- $11-5$

plll Viewed: 0 (pdf), 48 (html) Af Private Access

Effectiveness of Chlorogenic Acid Supplementation on VEGF Serum and Placental MAP Kinase Expression in Carbon Black-Exposed Pregnant Rattus norvegicus (AbstractView.aspx?PID=2018-11-5-25)

Viski Fitri Hendrawan, Desi Wulansari, Yudit Oktanella, Widjiati

DOI: $10.5958 / 0974-360 \times .2018 .00340$.

[Abstract] [PDF Paper] [HTML Paper] (HTMLPaper.aspx? Journal=Research Journal of Pharmacy and

Technology:PID=2018-11-5-25)

fill Viewed: 0 (pdf), 69 (html)

Effectiveness of Three Dimensional Approach of Schroth Method and Yoga on Pulmonary Function Test and Posture in Upper Crossed Syndrome With Neck Pain-A double blinded study (AbstractView.aspx?PID=2018-115-26)

V. Rajalaxmi, Jiby Paul, M. Nithya, S. Chandra Lekha, B. Likitha

DOI: 10.5958/0974-360X.2018.00341.4

[Abstract] [PDF Paper] [HTML Paper] (HTMLPaper.aspx? Journal=Research Journal of Pharmacy and

Technology;PID=2018-11-5-26) [Cite] fll Viewed: 0 (pdf), 74 (html) Private Access

Impact of nano Chelated Iron, GA3 and Organic Fertilizer (Acadian) in Moringa leaves Content of $\alpha$-Tocopherol and Phytosterols (AbstractView.aspx?PID=2018-11-5-27)

Abdulameer Ali Yaseen Akhlass Mery Kadim

ADdul: 105958/0974-360x 201800342 (6)

[Abstract] [PDF Paper] [HTML Paper] (HTMLPaper.aspx? Journal=Research Journal of Pharmacy and

Technology;

pll Viewed: 0 (pdf), 51 (html) in Private Access

Assessment of Family Lifestyle and Eating Habits for Home Prevention in Hilla City (AbstractView.aspx? PID=2018-11-5-28)

Amean A. Yasir

DOI: 10.5958/0974-360X2018.00343.

[Abstract] [PDF Paper] [HTML Paper] (HTMLPaper.aspx? Journal=Research Journal of Pharmacy and

Technology; PID=2018-11-5-28

pll Viewed: 0 (pdf), 63 (html) if Private Access

The effect of Cool Dialysis on Pruritus and Fatigue in Hemodialysis Patients (AbstractView.aspx?PID=2018-11-

5-29)

Eun-Mi Ham, Ji-Hye Lim, Sul-Hee Lee

DOI: 105958 J0974-360X $201800344 X$

[Abstract] [PDF Paper] [HTML Paper] (HTMLPaper.aspx? Journal=Research Journal of Pharmacy and

TAbstract] [PDF Paper] [HTML Paper] (HTMLPaper.aspx? Journal=Research Journal of Pharmacy and
Technology;PID=2018-11-5-29) [Cite]

Green Synthesis of Silver Nanoparticles by Fungus Beauveria bassiana and their Characteristics (AbstractView.aspx?PID=2018-11-5-3)

Marwah Amer Qamandar, Maan Abdul Azeez Shafeeq

DOI: 10.5958/0974-360X.2018.00319.0

[Abstract] [PDF Paper] [HTML Paper] (HTMLPaper.aspx? Journal=Research Journal of Pharmacy and

Technology;PID=2018-11-5-3) [Cite]

pill viewed: 0 (pdf), 66 (html) if Private Access

Biofilm Inhibition Efficiency of Endophytic Fungi Isolated from Acacia nilotica against Oral Pathogens (AbstractView.aspx?PID=2018-11-5-30)

Meenambiga SS, Rajagopal K

DOI: $10.5958 / 0974-360 \times 201800345.1$

[Abstract] [PDF Paper] [HTML Paper] (HTMLPaper.aspx?Journal=Research Journal of Pharmacy and

Technology;PID=2018-11-5-30) [Cite] fill Viewed: 0 (pdf), 54 (html) Private Access

Decision Support System for Glaucoma Diagnosis using Optical Coherence Tomography Images (AbstractView.aspx?PID=2018-11-5-31)

Sathiya KG, S. Srinivasan, T. S. Sivakumaran

DOI: $10.5958 / 0974-360 X .2018 .00346 .3$

[Abstract] [PDF Paper] [HTML Paper] (HTMLPaper.aspx? Journal=Research Journal of Pharmacy and

Technology:PI

ll Viewed: 0 (pdf), 77 (html) Private Access 
Phytochemical analysis and isolation of endophytic bacteria from Bauhinia purpurea (AbstractView.aspx? $\mathrm{PID}=2018-11-5-32$ )

Swetha Sunkar, Akshaya. A, Aarthi. B,

[Abstract] [PDF Paper] [HTML Paper] (HTMLPaper.aspx? Journal=Research Journal of Pharmacy and

Technology;PID=2018-11-5-32) [Cite]

pll Viewed: 0 (pdf), 81 (html) Ait Private Access

A Dose related ADR of a Siddha Drug Karpoora Chindhamani Mathirai (KCM) (AbstractView.aspx?PID=2018-115-33)

Meena R, Sathiyarajeswaran P, RamaswamyRS

DOI: 10.5958/0974-360X.2018.00348.7

[Abstract] [PDF Paper] [HTML Paper] (HTMLPaper.aspx? Journal=Research Journal of Pharmacy and

Technology;PID=2018-11-5-33) [Cite]

flll Viewed: 0 (pdf), 53 (html) Af Private Access

Comparison of Ferric Sulphate and Calcium Hydroxide as A Pulpotomy Agent (AbstractView.aspx?PID=2018

11-5-34)

BL. Abiraamasti, Deepa Gurunathan

00349.9

[Abstract] [PDF Paper] [HTML Paper] (HTMLPaper.aspx?Journal=Research Journal of Pharmacy and

Technology;PID=2018-11-5-34) [Cite]

pll Viewed: 0 (pdf), 55 (html) Private Access

Evaluation of Antibacterial activity of Zinc oxide Nanoparticles against Biofilm Producing Methicillin Resistant Staphylococcus aureus (MRSA) (AbstractView.aspx?PID=2018-11-5-35)

S. S. M. Umamageswari, B. Manipriya, M. Kalyan

DOI: $105958 / 0974-360 \times 201800350$

[Abstract] [PDF Paper] [HTML Paper] (HTMLPaper.aspx? Journal=Research Journal of Pharmacy and

pill Viewed: 0 (pdf), 68 (html) Af Private Access

Studies on Bioactive Compounds and Therapeutic Potential of Terminalia chebula Seed Extract AbstractView.aspx?PID=2018-11-5-36)

V. Mohanasrinivasan, Saranya D, Sumitha V, Kalpana R, Nivetha. A

DO1: 105958/0974-360X.2018003517

[Abstract] [PDF Paper] [HTML Paper] (HTMLPaper.aspx? Journal=Research Journal of Pharmacy and

Technology;PID=2018-11-5-36) [Cite]

fll Viewed: 0 (pdf), 58 (html) A Private Access

The Analysis of Organizational Approaches in Drug Registration in the EU, Ukraine, Tajikistan, Turkmenistan and Uzbekistan (AbstractView.aspx?PID=2018-11-5-37)

Hanna Panfilova, Olesia Nemchenko, Liusine Simonian, Oksana Tsurikova

DOI: $10.5958 / 0974-360 \times .2018 .00352 .9$

[Abstract] [PDF Paper] [HTML Paper] (HTMLPaper.aspx? Journal=Research Journal of Pharmacy and

Technology;PID=2018-11-5-37) [Cite]

Tll Viewed: 0 (pdf), 87 (html) Af Private Access

Antibacterial and Antifungal Potential of Marine Streptomyces sp. VITAK1 derived Novel Compound Pyrrolidinyl-Hexadeca-Heptaenone by in Silico docking Analysis (AbstractView.aspx?PID=2018-11-5-38)

Abirami Mani, Lokesh Ravi, Kannabiran Krishnan

DOl: $10.5958 / 0974-360 X .2018 .00353 .0$

[Abstract] [PDF Paper] [HTML Paper] (HTMLPaper.aspx? Journal=Research Journal of Pharmacy and

Technology;PID=2018-11-5-38) [Cite]

pill Viewed: 0 (pdf), 58 (html) Af Private Access

Antidepressive effects of Gami-Shinkiwhanin Immobilization stressed aging Mice (AbstractView.aspx? PID=2018-11-5-39)

Sun-Young Park Ki-Bong Kim, Sang-Hyun Ahn, Ho-Hyun Kim

DO: $1059580974-360 \times 2018003542$

[Abstract] [PDF Paper] [HTML Paper] (HTMLPaper.aspx? Journal=Research Journal of Pharmacy and

Technology;PID=2018-11-5-39) [Cite]

fll Viewed: 0 (pdf), 48 (html) Af Private Access

Biodegradation of Organo Phosphorous Chlorpyrifos using Pseudomonas aeruginosa PF1 isolated from paddy field (AbstractView.aspx?PID=2018-11-5-4)

Muthukrishnan Lakshmipathy, S. V. Abirami, Thukkaram Sudhakar

Dor $1059580074-360 \times 2018003207$

[Abstract] [PDF Paper] [HTML Paper] (HTMLPaper.aspx? Journal=Research Journal of Pharmacy and

Technology;PID=2018-11-5-4) [Cite]

Fill viewed: 0 (pdf), 67 (html) Af Private Access

Quick and Efficient Method for Callus culture from stem disc tissue of Garlic (Allium sativum L.)

AbstractView.aspx?PID=2018-11-5-40) 
Panka, Rita Singh Majumdar, Muteba Ndiba Cynthic

DOI: 10.5958/0974-360X.2018.00355.4

[Abstract] [PDF Paper] [HTML Paper] (HTMLPaper.aspx? Journal=Research Journal of Pharmacy and

Technology:PID=2018-11-5-40)

fll Viewed: 0 (pdf), 87 (html) Of Private Access

Antibacterial Activity of Soil Actinomycetes against Fish and Shellfish Pathogens (AbstractView.aspx?

PID=2018-11-5-41)

Mohammed Ishaque Nabila, Krishnan Kannabiran

Dol: $10.5958 / 0974-360 \times .2018 .00356 .6$

[Abstract] [PDF Paper] [HTML Paper] (HTMLPaper.aspx?Journal=Research Journal of Pharmacy and

Technology:PID-2018-11-5-41) [Cite]

Fill Viewed: 0 (pdf), 59 (html) Af Private Access

Pyrimidines as Potent Antioxidant Agents (AbstractView.aspx?PID=2018-11-5-42)

K. Ishwar Bhat, Abhishek Kumar

DOI: 10.5958/0974-360X.2018.00357.8

[Abstract] [PDF Paper] [HTML Paper] (HTMLPaper.aspx?Journal=Research Journal of Pharmacy and

Technology;PID=2018-11-5-42) [Cite] Pill Viewed: 0 (pdf), 44 (html) Private Access

Bilateral Variation in Adolescents: Handedness, Arm Positioning and Thumb Positioning (AbstractView.aspx? PID=2018-11-5-43)

Susie Jeyalyn David, W. M. S. Johnson

DOI: 10.5958/0974-360X.2018.00358.X

[Abstract] [PDF Paper] [HTML Paper] (HTMLPaper.aspx? Journal=Research Journal of Pharmacy and

Technology;PID=2018-11-5-43) [Cite]

fill Viewed: 0 (pdf), 53 (html) Af Private Access

Physicochemical and Acute Toxicity Study of Gunja (Abrus precatorius) Pericarp (AbstractView.aspx?

PID=2018-11-5-44)

Dev Nath Singh Gautam

DOI: 10.5958/0974-360X.2018.00359.

[Abstract] [PDF Paper] [HTML Paper] (HTMLPaper.aspx? Journal=Research Journal of Pharmacy and

Technology;PID=2018-11-5-44) [Cite]

Fill viewed: 0 (pdf), 66 (html) Af Private Access

Classification of Medicines using Naive bayes Classifier (AbstractView.aspx?PID=2018-11-5-45)

Baisani Indraja, Annapurani K

A74-360X201800360.8

[Abstract] [PDF Paper] [HTML Paper] (HTMLPaper.aspx?Journal=Research Journal of Pharmacy and

Technology;PID=2018-11-5-45) [Cite]

pll viewed: 0 (pdf), 59 (html) 1 Private Access

Self-Assistance Devices for Asthma Patients using Android Application (AbstractView.aspx?PID=2018-11-5-46) Bestley Joe S, Gomathi T, Mafiin Shaby S, Pandian R

DOI: 105958/0974-360X201800361X

[Abstract] [PDF Paper] [HTML Paper] (HTMLPaper.aspx? Journal=Research Journal of Pharmacy and

fill Viewed: 0 (pdf), 80 (html)

Predicting Life time of Heart Attack Patient using Improved C4.5 Classification Algorithm (AbstractView.aspx? PID=2018-11-5-47)

R. Jothikumar, S. Susi, N. Sivakumar, P. S. Ramesh

DOl: 10.5958/0974-360X.2018.00362.1

[Abstract] [PDF Paper] [HTML Paper] (HTMLPaper.aspx? Journal=Research Journal of Pharmacy and

Technology;PID=2018-11-5-47) [Cite]

pll Viewed: 0 (pdf), 74 (html) An Private Access

In silico Evaluation of Non-Synonymous SNPs in IRS-1 Gene associated with type II Diabetes Mellitus. (AbstractView.aspx?PID=2018-11-5-48)

Apoorva Singh, Siddhant Singh, Anand Anbarasu

DOI: 10 5958/0974-360X201800363.3

[Abstract] [PDF Paper] [HTML Paper] (HTMLPaper.aspx? Journal=Research Journal of Pharmacy and

Technology;PID=2018-11-5-48) [Cite]

fll Viewed: 0 (pdf), 47 (html) An Private Access

Boiling Point of Alkanes and Alkenes - From Graph Eccentricity (AbstractView.aspx?PID=2018-11-5-49)

M. Yamuna, T. Divya

DOI: 10.5958/0974-360X.2018.00364.5

[Abstract] [PDF Paper] [HTML Paper] (HTMLPaper.aspx? Journal=Research Journal of Pharmacy and

Technology;PID=2018-11-5-49) [Cite] fll Viewed: 0 (pdf), 56 (html) Private Access

Computational Study on Receptors Related to Aggressive Conduct (AbstractView.aspx?PID=2018-11-5-5) 
Balasankar Karavadi, Shivashankari S.

[Abstract] [PDF Paper] [HTML Paper] (HTMLPaper.aspx? Journal=Research Journal of Pharmacy and

Technology;PID=2018-11-5-5) [Cite]

fll Viewed: 0 (pdf), 66 (html) Af Private Access

Determination of Essential oil component composition of common Sunflower Marginal Flowers (AbstractView.aspx?PID=2018-11-5-50)

Gontova, T. M., Sokolova, O. O., Kotov, A. G., Kutsenko, S. A., Mashtaler, V. v.

DOl: $10.5958 / 0974-360 X .2018 .00365 .7$

[Abstract] [PDF Paper] [HTML Paper] (HTMLPaper.aspx? Journal=Research Journal of Pharmacy and

Technology;PID=2018-11-5-50

Ill Viewed: 0 (pdf), 52 (html) Private Access

Effect of Eucalyptus extracts on the vegetative growth and yield of Hordeum vulgare plant

(AbstractView.aspx?PID=2018-11-5-51)

Samah Saleh AL-Shybany

DOI: $10.5958 / 0974-360 \times .2018 .00366 .9$

[Abstract] [PDF Paper] [HTML Paper] (HTMLPaper.aspx? Journal=Research Journal of Pharmacy and

Technology;PID=2018-11-5-51) [Cite]

Pyrazolines as Potent Antioxidant Agents (AbstractView.aspx?PID=2018-11-5-52)

K. Ishwar Bhat, Abhishek Kumar

Dol: $10.5958 / 0974-360 X .2018 .00367 .0$

[Abstract] [PDF Paper] [HTML Paper] (HTMLPaper.aspx? Journal=Research Journal of Pharmacy and

Fill Viewed: 0 (pdf), 53 (html) fie Private Access

Caffeine Estimation in Darjeeling District Tea Samples (AbstractView.aspx?PID=2018-11-5-53)

M. Muthukkaruppan, A. Nithya, P. Parthiban

DOI: $10.5958 / 0974-360 X .2018 .00368 .2$

[Abstract] [PDF Paper] [HTML Paper] (HTMLPaper.aspx? Journal=Research Journal of Pharmacy and

Technology;PID=2018-11-5-53) [Cite]

pill Viewed: 0 (pdf), 74 (html) Private Access

Ion Sensitive Floating in Situ Gel for Controlled Delivery of Famotidine and Domperidone Maleate for the Treatment of Gastro Oesophageal Reflux Disease (AbstractView.aspx?PID=2018-11-5-54)

Devika Jorapur, Nagesh C, Suma N, Chandrasekhara S, Sunil L Attimarad, Savitha Kengeri

DOl: 10.5958/0974-360X.2018.00369.4

[Abstract] [PDF Paper] [HTML Paper] (HTMLPaper.aspx? Journal=Research Journal of Pharmacy and

Technology;PID=2018-11-5-54) [Cite]

fll Viewed: 0 (pdf), 65 (html) At Private Access

A small cross sectional assessment of Physiological Health Status in relation to Anthropometric and CardioRespiratory Indices among Head-Supported Load Carrying Female Porters of Sikkim, India

(AbstractView.aspx?PID=2018-11-5-55)

Koushik Bhattachary, Reena Kulshrestha, Sharit Shekhar Barman, Debdatta Bhadui

Dol: $10.5958 / 0974-360 X .2018 .00370 .0$

[Abstract] [PDF Paper] [HTML Paper] (HTMLPaper.aspx? Journal=Research Journal of Pharmacy and

Technology;PID=2018-11-5-55) [Cite] ill Viewed: 0 (pdf), 181 (html) A Private Access

Evaluation of Anti Depressant Activity of Petroleum Ether Extract of Rosa centifolia in Swiss Albino Mice (AbstractView.aspx?PID=2018-11-5-56)

Ferdous A. Jabir, Sundus K. Hamzah

Dol: $10.5958 / 0974-360 X .2018 .00372 .4$

[Abstract] [PDF Paper] [HTML Paper] (HTMLPaper.aspx? Journal=Research Journal of Pharmacy and

Fill Viewed: 0 (pdf), 50 (html) Private Access

Removal of Methylene Blue from an Aqueous Solution using Plumbago zeylanica Leaves-Optimisation using Response Surface Methodology (RSM) (AbstractView.aspx?PID=2018-11-5-57)

P. Pallavi, P. King, Y. Prasanna Kumar

DOI: $10.5958 / 0974-360 X .2018 .00373 .6$

[Abstract] [PDF Paper] [HTML Paper] (HTMLPaper.aspx?Journal=Research Journal of Pharmacy and

Fill Viewed: 0 (pdf), 48 (html) A Private Access

Determination of Clopidogrel bisulphate using Drug Selective Membranes (AbstractView.aspx?PID=2018-11-5-

Oussama Mansour, Dania Nashed, Amir Alhaj Sakur

DOI: $10.5958 / 0974-360 X .2018 .00374 .8$

[Abstract] [PDF Paper] [HTML Paper] (HTMLPaper.aspx?Journal=Research Journal of Pharmacy and

Technology;PID=2018-11-5-58) [Cite]

fll Viewed: 0 (pdf), 77 (html) A Private Access 
Pharmacognostic Profile of Trigonella corniculata L. Seeds and effect of its Aqueous Extract on Growth Inhibition of Cancer Cells (AbstractView. aspx?PID=2018-11-5-59)

Pardeep Kaur, Sanjeev Kumar Kataria, Balbir Singh3, Saroj Arora

DOI: $105958 / 0974-360 \times 201800375 \times$

[Abstract] [PDF Paper] [HTML Paper] (HTMLPaper.aspx? Journal=Research Journal of Pharmacy and

Technology:PID-2018-11-5-59) [Cite]

pll Viewed: 0 (pdf), 73 (html) An Private Access

Detection of Hemolysin and Gelatinase in Uropathological Escherichia coli (AbstractView.aspx?PID=2018-11-56)

F. Mariyam Niyas, Gopinath P

DOI: 105958/0974-360X 201800322

[Abstract] [PDF Paper] [HTML Paper] (HTMLPaper.aspx? Journal=Research Journal of Pharmacy and

Technology; PID=2018-11-5-6) [Cite]

Fill Viewed: 0 (pdf), 47 (html) An Private Access

Differential Pulse Anodic Stripping Voltammetric Analysis of Selenium (IV) at a Gold Electrode Modified with O-Phenylenediamine- Nafion (AbstractView.aspx?PID=2018-11-5-60)

Abdul Aziz Ramadan, Hasna Mandil, Abdulrahman Shikh-Debes

DOI: 10.5958/0974-360X201800376,

[Abstract] [PDF Paper] [HTML Paper] (HTMLPaper.aspx? Journal=Research Journal of Pharmacy and

Technology;PID $2018-11-5-60)$ [Cite]

pill Viewed: 0 (pdf), 82 (html) At Private Access

Anti-MRSA activity of Actinomycetes isolated from Marine Soil sample of Ariyaman Beach, Tamil Nadu, India (AbstractView.aspx?PID=2018-11-5-61)

Krishnamoorthy Bhakyashree, Krishnan Kannabira

DOI: 10.5958/0974-360X.2018.00377.3

[Abstract] [PDF Paper] [HTML Paper] (HTMLPaper.aspx? Journal=Research Journal of Pharmacy and

Technology;PID=2018-11-5-61) [Cite] fill Viewed: 0 (pdf), 54 (html) Private Access

Formulation and Evaluation of Colon Specific Matrix Tablet of Metronidazole (AbstractView.aspx?PID=2018-

11-5-62)

Ajazuddin, Tripti Banjare, Amit Alexander, Palak Agrawal, Akansha Bhandarkar, Aditi Bhatt, Swapnil Gupta, Hemlata Sahu, Shradha Devi Diwedi, Pankaj Sahu, Siddharth Kumar Sahu, Pooja Yadav, Kailash Sahu, Deeksha Dewangan, Hemlata Thapa, Deepika, Sonam Soni, Mukesh Sharma, D. K. Tripathi

DOI: 10.5958/0974-360X.2018.00378.5

[Abstract] [PDF Paper] [HTML Paper] (HTMLPaper.aspx? Journal=Research Journal of Pharmacy and

Technology:PID-2018-11-5-62) [Cite]

pll Viewed: 0 (pdf), 58 (html) An Private Access

Lung Cancer Detection using Image Processing Technique (AbstractView.aspx?PID=2018-11-5-63)

Sindhu Priya. S, B. Ramamurthy

DOl: $10.5958 / 0974-360 X .2018 .00379 .7$

[Abstract] [PDF Paper] [HTML Paper] (HTMLPaper.aspx? Journal=Research Journal of Pharmacy and

Technology:PID =

$$
\text { fll Viewed: } 0 \text { (pdf), } 81 \text { (html) } 1 \text { Private Access }
$$

Evaluation of Covariates using Population Pharmacokinetics approach for dose Individualization of Levothyroxine in Patients with Post Thyroidectomy and 131I ablation Therapy (AbstractView.aspx?PID=201811-5-64)

Rajesh Kumar, Mukhyaprana Prabhu, Sreedhar R Pai, Vasudev Shenoy

DOI: 10.5958/0974-360X201800380.3

[Abstract] [PDF Paper] [HTML Paper] (HTMLPaper.aspx? Journal=Research Journal of Pharmacy and

Technology:PID=2018-11-5-64) [Cite]

plll Viewed: 0 (pdf), 58 (html) Af Private Access

Evaluation of Covariates using Population Pharmacokinetics approach for dose Individualization of Levothyroxine in Patients with Post Thyroidectomy and 131 l ablation Therapy (AbstractView.aspx?PID=201811-5-65)

Rajesh Kumar, Mukhyaprana Prabhu, Sreedhar R Pai, Vasudev Shenoy

Rajesh Kumar, Mukhyaprana Prabhu,

[Abstract] [PDF Paper] [HTML Paper] (HTMLPaper.aspx? Journal=Research Journal of Pharmacy and

pll viewed: 0 (pdf), 74 (html) At Private Access

Effect of Lead and Aluminium in the levels of Dopamine and Acetylcholine in the Brain Male Rats (AbstractView.aspx?PID=2018-11-5-66)

Wejdan M. Kadhem, Hanaa Enaya

Dol: 10.5958/0974-360X.2018.00381.5

[Abstract] [PDF Paper] [HTML Paper] (HTMLPaper.aspx?Journal=Research Journal of Pharmacy and Technology;PID=2018-11-5-66) [Cite] 
Quantitative Estimation of Phytoconstituents and in vitro Anthelmintic Assessment of leaf Extracts of Tagetes erecta Linn. (AbstractView.aspx?PID=2018-11-5-67)

Shambaditya Goswami, Ravindra Pal Sing

DOI: 10.5958/0974-360X.2018.00382.7

[Abstract] [PDF Paper] [HTML Paper] (HTMLPaper.aspx? Journal=Research Journal of Pharmacy and

Fill Viewed: 0 (pdf), 72 (html) Private Access

A Case Study - Mathematical Models for Drug Tolerance and Cancer Chemotherapy (AbstractView.aspx?

$\mathrm{PID}=\mathbf{2 0 1 8 - 1 1 - 5 - 6 8 )}$

M. Yamuna, K. Karthika

DOI: $10.5958 / 0974-360 X .2018 .00383 .9$

[Abstract] [PDF Paper] [HTML Paper] (HTMLPaper.aspx? Journal=Research Journal of Pharmacy and

fill Viewed: 0 (pdf), 51 (html) Private Access

Evaluation of the Gram-Negative Bacilli Causing Surgical-Site Infections and their Sensitivity to Antibiotics in Al- Mowasat Hospital, Damascus, Syria (AbstractView.aspx?PID=2018-11-5-69)

RanaAhmad

DOI: $10.5958 / 0974-360 \times .2018 .00384 .0$

[Abstract] [PDF Paper] [HTML Paper] (HTMLPaper.aspx? Journal=Research Journal of Pharmacy and

fll Viewed: 0 (pdf), 54 (html) Af Private Access

Factors of the Complicated Course of the Myocardial Infarction in Men and Women (AbstractView.aspx? PID=2018-11-5-7)

Gulnara Bedelbayeva, Zhannat Nurmakhanova, Murat Almukhambetov, Nazima Zarubekova, Ainur Nuftieva

Dol: 10.5958/0974-360X.2018.00323.2

[Abstract] [PDF Paper] [HTML Paper] (HTMLPaper.aspx? Journal=Research Journal of Pharmacy and

Technology;PID=2018-11-5-7) [Cite] fll Viewed: 0 (pdf), 61 (html) A Private Access

Hazards and Problems Facing Nurse at Hiwa Hospital in Slemani City, Kurdistan Region, Iraq (AbstractView.aspx?PID=2018-11-5-70)

Galawezh Jalal Salih, Sanaa H. Abdul Sahib

DOl: $10.5958 / 0974-360 X .2018 .00385 .2$

[Abstract] [PDF Paper] [HTML Paper] (HTMLPaper.aspx? Journal=Research Journal of Pharmacy and

Technology;PID 20

Formulation and Evaluation of Gastro Retentive Sustained Release Tablets of Ziprasidone Hydrochloride (AbstractView.aspx?PID=2018-11-5-71)

Kailash Sahu, Amit Alexander, Hemlata Thapa, Tripti Banjare, Palak Agrawal, Akansha Bhandarkar, Aditi Bhatt, Swapnil Gupta, Hemlata Sahu, Shradha Devi Diwedi, Pankaj Sahu, Siddharth Kumar Sahu, Pooja Yadav, Deeksha Dewangan, Deepika, Hemant Ramchandra Badwaik, Mukesh Sharma, D. K. Tripathi, Ajazuddin

DOI: 10.5958/0974-360X.2018.00386.4

[Abstract] [PDF Paper] [HTML Paper] (HTMLPaper.aspx? Journal=Research Journal of Pharmacy and

Fill Viewed: 0 (pdf), 45 (html) Private Access

HPLC determination of Sildenafil Tartrate and its related Substances along with some Supportive Studies using MS, XRD and NMR (AbstractView. aspx?PID=2018-11-5-72)

O.S.S. Chandana, D. Swapna, R. Ravichandra Babu

DOI: 10.5958/0974-360X.2018.00387.6

[Abstract] [PDF Paper] [HTML Paper] (HTMLPaper.aspx? Journal=Research Journal of Pharmacy and

Technology;PID=2018-11-5-72) [Cite] fll Viewed: 0 (pdf), 91 (html) Private Access

Sample Microwave Digestion for the Determination of Manganese, Iron, Zinc, Copper and Nickel in Catfish Consumed in Syria by Flame Atomic Absorption Spectrometry (AbstractView.aspx?PID=2018-11-5-73)

Saad Antakli, Nazira Sarkis, Firas Nahas

DOI: $10.5958 / 0974-360 X .2018 .00388 .8$

[Abstract] [PDF Paper] [HTML Paper] (HTMLPaper.aspx?Journal=Research Journal of Pharmacy and

Fill Viewed: 0 (pdf), 59 (html) A Private Access

Ion Sensitive Floating In Situ gel for Controlled Delivery of Famotidine and Domperidone Maleate for the Treatment of Gastro Oesophageal Reflux Disease (AbstractView.aspx?PID=2018-11-5-74)

Devika Jorapur, Nagesh C, Suma N, Chandrasekhara S, Sunil L Attimarad, Savitha Kenger

DOI: $10.5958 / 0974-360 X .2018 .00389 . x$

[Abstract] [PDF Paper] [HTML Paper] (HTMLPaper.aspx?Journal=Research Journal of Pharmacy and

fill viewed: 0 (pdf), 58 (html) Af Private Access 
Prevalence of Uropathogens among Diabetic Patients in Punjab, India (AbstractView.aspx?PID=2018-11-5-75)

Ashish Vyas, Karamjeet Singh, Gaurav Kumar

[Abstract] [PDF Paper] [HTML Paper] (HTMLPaper.aspx? Journal=Research Journal of Pharmacy and

Technology;PID=2018-11-5-75) [Cite]

fll Viewed: 0 (pdf), 60 (html) An Private Access

Role of Neprilysin Inhibitors in Cardiovascular Disease- A Short Review (AbstractView.aspx?PID=2018-11-5-76) Jayashree K.A

DOI: $10.5958 / 0974-360 X .2018 .00391 .8$

[Abstract] [PDF Paper] [HTML Paper] (HTMLPaper.aspx? Journal=Research Journal of Pharmacy and

Technology;PID=2018-11-5-7

Ill Viewed: 0 (pdf), 57 (html) Aprivate Access

Determination of Wiener Polarity Index from Reduced Distance Matrix and Molecular Structure of Drugs - A

Theoretical Approach (AbstractView.aspx?PID=2018-11-5-77)

M. Yamuna, T. Divya

DOI: 10.5958/0974-360X.2018.00392X

[Abstract] [PDF Paper] [HTML Paper] (HTMLPaper.aspx? Journal=Research Journal of Pharmacy and

Technology:PID=2018-11

Nill Viewed: 0 (pdf), 54 (html) Private Access

A Systematic Review on the Effect of Use of Impregnated Retraction Cords on Gingiva (AbstractView.aspx? PID=2018-11-5-78)

Abinaya Kannan, Suresh Venugopalan

DOl: 10.5958/0974-360X.2018.00393.1

[Abstract] [PDF Paper] [HTML Paper] (HTMLPaper.aspx? Journal=Research Journal of Pharmacy and

Fetor Oris - A Review on Classification and Management (AbstractView.aspx?PID=2018-11-5-79)

K. Siva Priyah, Sherlyn Sheeba, Dhanraj Ganapathy

DOI: $10.5958 / 0974-360 X .2018 .00394 .3$

[Abstract] [PDF Paper] [HTML Paper] (HTMLPaper.aspx? Journal=Research Journal of Pharmacy and

fill Viewed: 0 (pdf), 44 (html) At Private Access

The effects of SM-215 on androgeneticalopecia (AbstractView.aspx?PID=2018-11-5-8)

Sun-Young Park, Ki-Bong Kim, Sang-Hyun Ahn, Ho-Hyun Kim

DOI: 10.5958/0974-360X.2018.00324.

[Abstract] [PDF Paper] [HTML Paper] (HTMLPaper.aspx? Journal=Research Journal of Pharmacy and

Technology:PID=2018-11-5-8) [Cite]

fll viewed: 0 (pdf), 61 (html) Af Private Access

A Review on Medicinal Properties of Zanthoxylum armatum DC (AbstractView.aspx?PID=2018-11-5-80)

Hayat M. Mukhtar, Vandna Kalsi

DOl: 105958/0974-360X2018.00395.5

[Abstract] [PDF Paper] [HTML Paper] (HTMLPaper.aspx? Journal=Research Journal of Pharmacy and

Technology;PID=2018-11-5-80) [Cite]

pill Viewed: 0 (pdf), 50 (html) Af Private Access

Analytical Methods of Ciprofloxacin and its Combinations Review (AbstractView.aspx?PID=2018-11-5-81)

Ghoufran Kawas, Mohammad Marouf, Oussama Mansour, Amir Alhaj Sakur

DO1: $105958 / 0974-360 \times 2018.00396 .7$

[Abstract] [PDF Paper] [HTML Paper] (HTMLPaper.aspx? Journal=Research Journal of Pharmacy and

Technology;PID=2018-11-5-81) [Cite]

ell Viewed: 0 (pdf), 72 (html) A. Private Access

A Review on the Role of Presenilin in Alzheimer's Disease (AbstractView.aspx?PID=2018-11-5-82)

M. Vijey Aanandhi, Yeshwanth Prasanna Kumar. B, Ranadheer Chowdary. P, Praveen. D

M. Vijey $10.5958 / 0974-360 X$.2018.00397.9

[Abstract] [PDF Paper] [HTML Paper] (HTMLPaper.aspx? Journal=Research Journal of Pharmacy and

Technology;PID=2018-11-5-82) [Cite]

Pll Viewed: 0 (pdf), 53 (html) A. Private Access

The Levels of Vitamin E in Seminal Fluids and their association with Male Infertility: Short Review (AbstractView.aspx?PID=2018-11-5-83)

Abdulsamie H. Alta'ee, Hanan Abbas Majeed, Mahmoud H. Hadwa

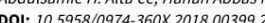

[Abstract] [PDF Paper] [HTML Paper] (HTMLPaper.aspx? Journal=Research Journal of Pharmacy and

Technology; PID $=2018-11-5-83$ )

fll viewed: 0 (pdf), 79 (html) in Private Access 


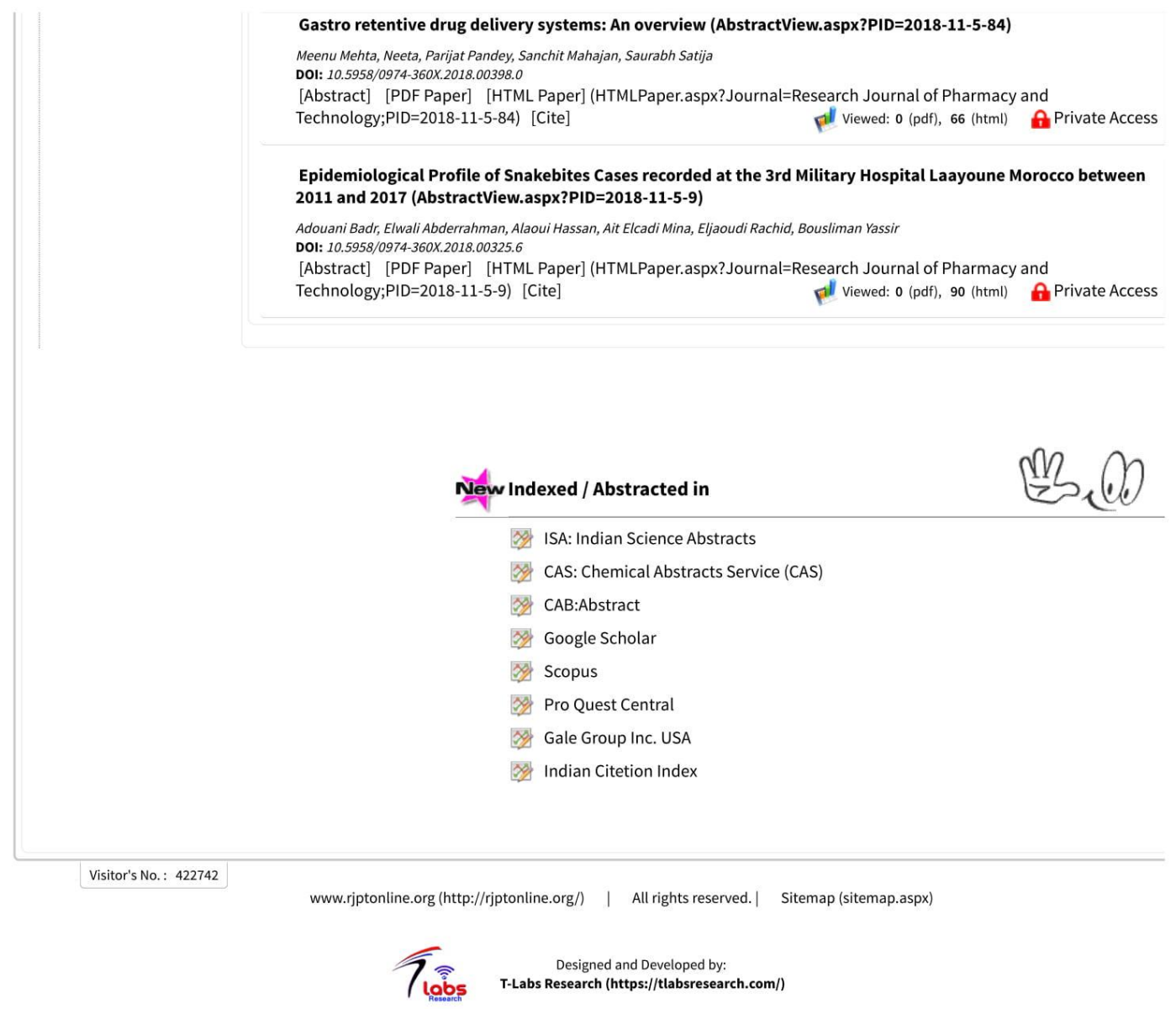


Editor :Research Journal of Pharmacy and Technology

RJPT House, Lokmanya Grih Nirman Society,

Rohanipuram, In-front of Sector- 1,

Pt. Deendayal Upadhyay Nagar

Raipur - 492010 Chhattisgarh, India

Publisher :A and V Publication

\section{Alamat web:}

http://rjptonline.org/HTMLPaper.aspx?Journal=Research\%20Journal \%20of\%20Pharmacy\%20and\%20Technology;PID=2018-11-5-25

DOI: $10.5958 / 0974-360 X .2018 .00340 .2$

Volume No. : 11

Issue No. : 5

Year : 2018

ISSN Print : 0974-3618

ISSN Online : 0974-360X 


\title{
Effectiveness of Chlorogenic Acid Supplementation on VEGF Serum and Placental MAP Kinase Expression in Carbon Black- Exposed Pregnant Rattus norvegicus
}

\author{
Viski Fitri Hendrawan ${ }^{1}$, Desi Wulansari ${ }^{1}$, Yudit Oktanella1, Widjiati ${ }^{2}$ \\ ${ }^{1}$ Department of Veterinary Reproduction, Faculty of Veterinary Medicine, Brawijaya University, Malang \\ ${ }^{2}$ Department of Anatomy, Faculty of Veterinary Medicine, University of Airlangga, Surabaya \\ *Corresponding Author E-mail: viski_hendrawan88@yahoo.com
}

\begin{abstract}
:
The aim of this study was to determine the effect of chlorogenic acid on the decrease of placental apoptosis in pregnant Rattus norvegicus exposed to carbon black by observing the expression of Mitogen Activating Protein Kinase (MAP Kinase) and Vascular Endhotel Growth Factor (VEGF) as indicators of apoptosis. This study was a laboratory experimental study, with five treatment groups and seven replications using female rats (Rattus norvegicus). The study was started by mating the female rats and exposing them to carbon black of $1064 \mathrm{mg} / \mathrm{m} 3$ for 8 hours. Then, for MAP Kinase expression examination, immunohistochemical staining was performed and VEGF measurements were done using ELISA. Administration of chlorogenic acid at a dose of 13.4 $\mathrm{mg} / \mathrm{kg}$ body weight in pregnant rats exposed to carbon black resulted in a decrease of MAP Kinase expression in placenta compared with a positive control group not exposed to carbon black. Carbon black-exposed pregnant rats treated with chlorogenic acid at a dose of $13.4 \mathrm{mg} / \mathrm{kg} \mathrm{BW}$ showed increased VEGF levels compared with a positive control group not exposed to carbon black. In conclusion, the administration of chlorogenic acid may decrease MAP Kinase and increase VEGF levels.
\end{abstract}

KEYWORDS: Carbon black; MAP Kinase; VEGF; Rattus norvegicus.

\section{INTRODUCTION:}

Carbon black is a cytotoxic, genotoxic and most stable particulate matter (PM) in the air in the form of carbon monoxide (CO) compounds. The amount of CO in the air is close to $164.383,56 \mathrm{mg} / \mathrm{m} 3$ every day. This carbon source comes from factory exhaust gas, motor vehicle emissions, and domestic waste incineration. Some individuals may be exposed to the presence of carbon black in the working environment. Communities most frequently exposed to carbon black are factory workers, motor vehicle workshops, fuel industry, oil and coal industries.

The association of carboxyhemoglobin causes tissue and placenta hypoxia by expressing Hypoxia-Induced Factor-1 Alpha (HIFlalpha) in order to decrease angiogenesis processes that may lead to impaired growth and development in the fetus, preterm birth and low birth weight (Rifatul, 2011) According to Gratton (2001), VEGF is one of the angiogenic cytokines secreted by various cells and serves as a survival factor for endothelial cells in vivo and in vitro. VEGF may also act as a mitogen and chemoattractant. In the process of angiogenesis, VEGF plays a role to repair damaged blood vessels, as well as for the process of feeding the fetus that passes through the blood vessels. Increased MAP Kinase p53 will cause disruption of the placenta, whose implementation will lead to premature birth. Increased cytokines may interfere with the differentiation of ectoderm, mesoderm and endoderm cells during the course of the pro-inflammatory organogenesis process. Disturbance of mesoderm cell differentiation may cause disturbance to bone growth (Gilbert, 1988). Antioxidant provision is one way to suppress free radicals. Coffee contains one substance that acts as an antioxidant. Green coffee beans (Coffee robusta Lindau.) are rich in polyphenols. Tannin is the main polyphenol content of coffee beans, while chlorogenic acid (CGA) is the main polyphenol in coffee beans. CGA content in arabica coffee is $4-8.4 \%$ and in robusta coffee is $7-14.4 \%$ (Thom, et al., 2007). According to EFSA (2011), the dose of CGA to prevent the occurrence of DNA damage, prevent apoptosis, and stabilize blood glucose levels of $9 \mathrm{mg} / \mathrm{kg}$ body weight. This study aims to determine the effective dose and antioxidant role contained in Chlorogenic Acid (CGA) in Green coffee bean (Coffea robusta Lindau) in improving body weight and fetal length, blood VEGF levels, and MAP Kinase expression.

\section{MATERIALS AND METHODS:}




\section{Research procedure:}

\section{In vivo pregnancy induction:}

The rats were injected with Pregnant Mare Serum Gonadotropin (PMSG) of 10 IU, then after 48 hours, injected with 10 IU of Human Chorionic Gonadotropin (HCG). Mating with male rats was performed with monomating method between one male rat and one female rat in a cage. Seventeen hours after being mated, the vaginal plug was examined. If the presence of vaginal plug was positive, the rat was considered as being pregnant on day zero and collected as a population of pregnant rats. If no vaginal plug was found, it was considered negative and not used as a sample.

Selection of carbon black:

Carbon black is carbon in powder form which has been widely used as a model for particles diesel emission without chemistry and metals. Some its chemical and physical features are similar to nano-based carbon engineering particles. Carbon black comprises carbon with impurities less than $1 \%$ organic and inorganic (Brown et al., 2000).

\section{Population and sample:}

This study was a true experimental laboratory study with Randomized Complete Randomized Design model which used rats consisting of 5 groups, with the number of replications per group of 6 rats each, with the following calculation: $t(n-1)$ ? 15; with $\mathrm{t}=$ number of treatments, $\mathrm{n}=$ number of replications (Kusriningrum, 2000). The negative control group comprised 6 normal rats without treatment $(\mathrm{KN})$; positive control group: pregnant rats+receiving carbon black treatment and dissected on day 18 (KP); treatment group 1: 6 pregnant rats+carbon black exposure at $1064 \mathrm{mg} / \mathrm{m} 3$ for 8 hours per day, at 6-17 days gestation and sacrifice on day $18+$ receiving choolorogenic acid supplementation at $6-17$ day gestation $4.5 \mathrm{mg} / \mathrm{kg}$ body weight (P1); and treatment group 2: 6 pregnant rats+carbon black exposure at $1064 \mathrm{mg} / \mathrm{m} 3$ dose for 8 hours per day at 6-17 days gestation, and sacrificed on day 18+ receiving cholorogenic acid supplementation at 6-17 days gestation of $9 \mathrm{mg} / \mathrm{kg}$ body weight (P2); treatment group 3: 6 pregnant rats + carbon black exposure at $1064 \mathrm{mg} / \mathrm{m} 3$ dose for 8 hours per day at 6-17 days gestation and sacrificed on day 18+, receiving cholorogenic acid supplementation at $6-17$ days gestation by $13.5 \mathrm{mg} / \mathrm{kg}$ body weight (P3).

\section{Measurements of serum VEGF levels with ELISA:}

All reagents were left at room temperature $\left(18-25^{\circ} \mathrm{C}\right)$ before use. Pipetted as much as 50 ?L standard, the samples were inserted into the microplate. A $100 \mu \mathrm{l}$ VEGF Enzym Conjugate was added for each microplate, then shaken for 30 seconds and incubated at $37^{\circ} \mathrm{C}$ for 1 hour. After incubation, the solution on the microplate was removed and then washed with a Washing Solution 250 300 ?L and shaking were performed for 3 minutes. Washing was repeated for 5 times. Then the solution was reversed, pressed firmly with absorbent paper and dried with a tissue. A $100 \mu 1$ substrate of TBM solution was added to each microplate according to the sequence. The tube was incubated for 10 minutes at room temperature and covered with film glass and then wrapped with aluminum foil. To stop the reaction, 50 "l Stop Solution was added to each microplate gently. The mix was ?? shaken for 5 seconds. Then the microplate was fed into the ELISA Spectrophotometer, read and observed (Farah et al., 2006).

Examination of MAP kinase expression in placenta with immunohistochemistry:

The identification procedure of MAP kinase expression was performed by the immunohistochemical method in which the placenta preparations prepared on the glass object were dyed in xylol twice, stratified alcohols $(100 \%, 90 \%, 80 \%, 70 \%$ and $30 \%)$ respectively, then washed in PBS with a pH of 7.43 times each for 5 min, immersed in 3\% hydrogen peroxidase (H2O2) for 5-10 min, and immersed in 1\% BSA in PBS for 10-30 min at room temperature. The primary antibody of MAP Kinase was added for 1 hour at room temperature, washed in PBS ph 7.4 for 3 times 5 minutes. SA-HRP (Strep Avidin-Horse Radish Peroxidase) was added for 30-60 minutes at room temperature, and washed in PBS pH 7.4 for $3 \times 5$ minutes. Chromogen DAB $(3,3-$ diaminobenzidine tetrahydrochloride) was added for 10-20 min and washed in PBS for 3 times $5 \mathrm{~min}$ at room temperature, then counterstain was added using methyl green for $3 \mathrm{~min}$. Mounting was done with entellan. Observations were made using a microscope at 1000 magnification times. Determination of the amount of MAP Kinase expression was determined from the amount of brownish color change in placenta cells compared with the controls (Robyt and White, 1987; Aulanni'am 2004).

\section{RESULTS AND DISCUSSION:}

\section{MAP kinase expression on the placenta:}

MAP Kinase expression in each sample was assessed semiquantitatively according to modified Remmele method (Novak et al., 2007). Remmele scale index (IRS) is the multiplication of immunoreactive cells percentage score that expresses MAP kinase with color intensity score generated on cells. The effect of carbon black exposure on placental MAP Kinase expression through immunohistochemical staining based on the dose of chlorogenic acid therapy was calculated semi quantitatively, and the analysis of MAP Kinase expression was done using Kruskal Wallis method, which can be seen in Table 1.

Table 1. Expression of MAP kinase in exposed pregnant rat placenta

Table 1. Expression of MAP kinase in exposed pregnant rat placenta
\begin{tabular}{|l|l|l|l|l|}
\hline Treatment & $\begin{array}{l}\text { Positive } \\
\text { percentage score } \\
\text { (A) }\end{array}$ & $\begin{array}{l}\text { Color intensity } \\
\text { score (B) }\end{array}$ & Irs Index (AxB) & $\begin{array}{l}\text { Mean Rank } \pm \\
\text { Standard Deviation }\end{array}$ \\
\hline
\end{tabular}




\begin{tabular}{|c|c|c|c|c|c|}
\hline $\begin{array}{l}\text { KN } \\
\text { Pregnant rats without } \\
\text { carbon black exposure }\end{array}$ & $\begin{array}{l}\mathrm{KN}-1 \\
\mathrm{KN}-2 \\
\mathrm{KN}-3 \\
\mathrm{KN}-4 \\
\mathrm{KN}-5 \\
\mathrm{KN}-6 \\
\mathrm{KN}-7\end{array}$ & $\begin{array}{l}2 \\
1 \\
0 \\
0 \\
1 \\
1 \\
1\end{array}$ & $\begin{array}{l}1 \\
1 \\
0 \\
0 \\
0 \\
1 \\
1\end{array}$ & $\begin{array}{l}2 \\
1 \\
0 \\
0 \\
0 \\
1 \\
1\end{array}$ & $7.36 \pm 0.65^{\mathrm{a}}$ \\
\hline $\begin{array}{l}\text { KP } \\
\text { carbon black exposure } \\
1064 \mathrm{mg} / \mathrm{m}^{3} \text { to pregnant } \\
\text { rats aged } 6-17 \text { days for } 8 \\
\text { hours/day }\end{array}$ & $\begin{array}{l}\text { KP-1 } \\
\text { KP-2 } \\
\text { KP-3 } \\
\text { KP-4 } \\
\text { KP-5 } \\
\text { KP-6 } \\
\text { KP-7 }\end{array}$ & $\begin{array}{l}3 \\
3 \\
2 \\
2 \\
1 \\
1 \\
2\end{array}$ & $\begin{array}{l}3 \\
3 \\
3 \\
3 \\
3 \\
3 \\
1\end{array}$ & $\begin{array}{l}9 \\
9 \\
6 \\
6 \\
3 \\
3 \\
2 \\
\end{array}$ & $28.50 \pm 1.17^{b}$ \\
\hline $\begin{array}{l}\text { P1 } \\
\text { carbon black exposure } \\
1064 \mathrm{mg} / \mathrm{m} 3 \text { to pregnant } \\
\text { rats aged } 6-17 \text { days for } 8 \\
\text { hours } / \text { day }+ \text { CGA dose } \\
4.5 \mathrm{mg} / \mathrm{kg} \mathrm{BW}\end{array}$ & $\begin{array}{l}\text { P1-1 } \\
\text { P1-2 } \\
\text { P1-3 } \\
\text { P1-4 } \\
\text { P1-5 } \\
\text { P1-6 } \\
\text { P1-7 }\end{array}$ & $\begin{array}{l}2 \\
3 \\
3 \\
3 \\
1 \\
3 \\
2 \\
\end{array}$ & $\begin{array}{l}3 \\
3 \\
2 \\
2 \\
3 \\
1 \\
1\end{array}$ & $\begin{array}{l}6 \\
9 \\
6 \\
6 \\
3 \\
3 \\
2 \\
\end{array}$ & $27.93 \pm 1.35^{b c}$ \\
\hline $\begin{array}{l}\text { P2 } \\
\text { carbon black exposure } \\
1064 \mathrm{mg} / \mathrm{m}^{3} \text { to pregnant } \\
\text { rats aged } 6-17 \text { days for } 8 \\
\text { hours/day }+ \text { CGA dose } 9 \\
\mathrm{mg} / \mathrm{kg} \mathrm{BW}\end{array}$ & $\begin{array}{l}\text { P2-1 } \\
\text { P2-2 } \\
\text { P2-3 } \\
\text { P2-4 } \\
\text { P2-5 } \\
\text { P2-6 } \\
\text { P2-7 } \\
\end{array}$ & $\begin{array}{l}1 \\
0 \\
0 \\
1 \\
2 \\
1 \\
1\end{array}$ & $\begin{array}{l}3 \\
0 \\
0 \\
2 \\
1 \\
2 \\
1\end{array}$ & $\begin{array}{l}3 \\
0 \\
0 \\
2 \\
2 \\
2 \\
1 \\
\end{array}$ & $17.50 \pm 0.68^{c}$ \\
\hline $\begin{array}{l}\text { P3 } \\
\text { carbon black exposure } \\
1064 \mathrm{mg} / \mathrm{m}^{3} \text { to pregnant } \\
\text { rats aged 6-17 days for } 8 \\
\text { hours } / \text { day }+ \text { CGA dose } \\
13.5 \mathrm{mg} / \mathrm{kg} \mathrm{BW}\end{array}$ & $\begin{array}{l}\text { P3-1 } \\
\text { P3-2 } \\
\text { P3-3 } \\
\text { P3-4 } \\
\text { P3-5 } \\
\text { P3-6 } \\
\text { P3-7 }\end{array}$ & $\begin{array}{l}1 \\
0 \\
1 \\
1 \\
0 \\
1 \\
0 \\
\end{array}$ & $\begin{array}{l}2 \\
0 \\
1 \\
1 \\
0 \\
3 \\
0 \\
\end{array}$ & $\begin{array}{l}2 \\
0 \\
1 \\
1 \\
0 \\
3 \\
0 \\
\end{array}$ & $8.71 \pm 0.71^{\mathrm{a}}$ \\
\hline
\end{tabular}

KN: Negative control, pregnant rats without exposure to carbon black and without Chlorogenic Acid therapy

KP: Positive control, pregnant rats exposed to carbon black without Chlorogenic Acid therapy

P1: Treatment group 1, pregnant rats exposed to carbon black with Chlorogenic Acid therapy at $4.5 \mathrm{mg} / \mathrm{kg} \mathrm{BW}$

P2: Treatment group 2, pregnant rats exposed to carbon black with Chlorogenic Acid therapy at $9 \mathrm{mg} / \mathrm{kg} \mathrm{BW}$

P3: Treatment group 3, pregnant rats exposed to carbon black with Chlorogenic Acid therapy at $13.5 \mathrm{mg} / \mathrm{kg} \mathrm{BW}$

Notation:

$a, b, c$ and $d$ : difference of notation showed a significant difference between treatment groups $(p<0.05)$

Table 1 shows that MAP Kinase expression in the negative control group (a group of pregnant rats without treatment of carbon black and without Chlorogenic Acid therapy) did not show significant results compared with P3 group (a group of pregnant rats exposed to carbon black, with Chlorogenic Acid therapy of $13.5 \mathrm{mg} / \mathrm{kg} \mathrm{BW}$ ) as indicated by a low percentage of MAP Kinase immunoreactive cell. Positive control group (a group of pregnant rats exposed to carbon black without Chlorogenic Acid therapy) and P1 group (pregnant rats, exposed to carbon black, and Cholorogenic Acid therapy of $4.5 \mathrm{mg} / \mathrm{kg}$ BW) showed higher immunoreactive cell percentage scores than negative controls, P3 and P2. P2 group (pregnant rats, with carbon black exposure, and Cholorogenic Acid therapy of $9 \mathrm{mg} / \mathrm{kg} \mathrm{BW}$ ) showed MAP Kinase immuneoreactive cell percentage score lower than positive control group and P1, but MAP Kinase immunoreactive cell percentage score in P2 was higher than those in P3 and negative control.

Low MAP Kinase immunoreactive score in placenta shows the intensity of light brown color in immunoreactive placenta cells. High immunoreactive MAP Kinase score in placenta shows dark brown. The expression placenta MAP Kinase in various treatment groups can be seen in Figure 1.

Figure 1. Differences in MAP Kinase expression on trophoblast cells (arrows) of placenta between treatments.

The above slide shows that MAP Kinase expression in P3 group is weaker than those in other treatment groups (KP, P1, and P2). The above slide also shows that the P3 immunoreactive cell expression was almost the same as that in the negative control group (immunohistochemical staining, 1000x magnification, Nikon H600L microscope, DS Fi2 300 megapixel camera).

Carbon black is the most widely used nanomaterial and an exhaust gas of motor vehicles, which is cytotoxic and genotoxic. Carbon black is known to affect the fetus through several mechanisms, including directly entering the placenta, causing a change in placental function, and indirectly affecting the cytokine changes present in the placenta through the respiratory process 
(Hougraad et al., 2011).

Exposure to carbon black in pregnant rats may lead to the intrauterine fetus to produce carbon monoxide in the body with higher levels. The high exposure to carbon monoxide produced by carbon black during the burning process may increase the bond between hemoglobin and carbon monoxide that produces carboxyhaemoglobin. The binding of carboxyhemoglobin may cause tissue and placental hypoxia by expressing Hypoxia Induced Factor-1 Alpha (HIF-1alpha). Exposure to hydrocarbons affects the morphological and physiological responses of the rats placenta, especially in placental labyrinthine, the site of encounter of parental and fetal blood circulation. The presence of hydrocarbon exposure causes high ROS in placental cells, including endothelial blood vessels. High ROS causes both necrosis and apoptosis in endothelial cells. At placental level, oxidative stress occurring during pregnancy will induce macrophages to produce pro-inflammatory cytokines by modulating the transcription of pro-inflammatory genes through a phosphorylation cascade using MAP Kinase.

Chlorogenic acid is an antioxidant that inhibits increased ROS in tissues and prevents tissue hypoxia, thereby directly decreasing MAP Kinase expression in placenta. Administration of chlorogenic acid therapy at $13.5 \mathrm{mg} / \mathrm{kg}$ BW may decrease MAP Kinase expression in order to prevent excessive placental apoptosis, to prevent premature abortion and to improve the performance of pregnant rats.

\section{VEGF levels:}

Cytotoxic and genotoxic carbon black exposure may affect the angiogenesis process in the placenta of pregnant rats during pregnancy of 6-17 days and will eventually lead to increased apoptosis. The analysis used on the VEGF level was carried out in a depressive manner. Table 2 shows the mean and standard deviations in various treatment groups (KN, KP, P1, P2, and P3).

Table 2. VEGF levels in pregnant rats exposed to carbon black without and with chlorogenic acid therapy

\begin{tabular}{|l|l|}
\hline Groups & Mean \pm Standard Deviation \\
\hline Control Negative & $192.33 \pm 14.84$ \\
\hline Control Positive & $162 \pm 36.98$ \\
\hline Treatment 1 & $231 \pm 36.94$ \\
\hline Treatment 2 & $249 \pm 36.4$ \\
\hline Treatment 3 & $263.16 \pm 21.65$ \\
\hline
\end{tabular}

Table 2 shows the mean difference between treatments. The lowest mean VEGF level was shown in the positive control group (KP), while the highest mean in treatment group 3 (P3) that received carbon black exposure and CGA supplementation in a dose of $13.5 \mathrm{mg} / \mathrm{Kg}$ BW. This can also be seen in Figure 2.

Figure 2. VEGF in pregnant rats exposed to carbon black without and with chlorogenic acid therapy.

Figure 2 shows high VEGF levels in P3, P2, P1, and KN compared to KP. This suggests that chlorogenic acid has an effect on the increase of VEGF levels, which indicates that high levels of VEGF are able to regenerate in the blood vessel system to prevent apoptosis. Carbon black exposure given to pregnant rats causes cytokine changes in the placenta that will affect cellular response. This condition will contribute to inflammation and disruption of homeostasis tissue, which will continuously affect the vascular system in the placenta. Endothelial blood vessel cells, located along the vascular tract in the placenta, are impaired. As a result of inflammation, existing cytokines will contribute to pathological processes, such as angiogenesis, vascular homeostasis, and ischemia (Monique et al., 2001)

VEGF acts as a pro-inflammatory cytokine by increasing endothelial cell permeability, stimulating endothelial expression, molecular adhesion, and having the ability to act as a monocyte chemoattractant. VEGF is widely expressed by epidermal keratinocytes cells for wound healing and psoriasis, characterized by increased microvascular permeability and angiogenesis. One of the most influential cytokines in the formation of new blood vessels is VEGF. There are many factors that affect VEGF expression, one of which is the hypoxic stimulus (Levy et al., 1995). Other factors, such as cytokines, oncogenes, NO, and protein modulators kinase c, were reportedly capable of stimulating VEGF production (Brown et al., 1997). It was also reported that MAP Kinase and phosphatidylinositol-3-kinase (PI3-kinase) are involved in VEGF induction by growth factors, cytokines, and hypoxia (Clarke et al., 2001; Jiang et al., 2000).

In pregnant rats exposed to carbon black, the vascularization regeneration system of the rat placenta is quite high and is functioned to withstand the inflammatory invasion, since rats' placenta type has highly complex branching in the placental labyrinth area with the intention that the exchange of nutrition between the mother and the fetus is efficient (Watson and Cross, 2005). Pregnant rats exposed to carbon black and supplemented with Chlorogenic Acid in a dose of $13.5 \mathrm{mg} / \mathrm{kg}$ showed a good regeneration of 
vascularization with an indication of high levels of VEGF that resist inflammatory invasion, while maintaining nutrient exchange, and preventing excessive apoptosis in the placenta at the early age of pregnancy so that it can prevent the occurrence of early abortion.

\title{
CONCLUSION:
}

The administration of Chlorogenic Acid at a dose of $13.4 \mathrm{mg} / \mathrm{kg}$ body weight in pregnant rats exposed to carbon black may decrease MAP Kinase expression and increase VEGF levels in the placenta compared with positive control group not receiving Chlorogenic Acid.

\section{ACKNOWLEDGMENT:}

Research and Development Center, Ministry of Health, Republic of Indonesia, Dr. Widjiati, drh.,MS. Dan Prof. Dr. Aulani'am, drh., DES. dan Dr. Agung Praman Warih Marhendra, MS.

\section{REFERENCES:}

1 European Food Safety Authority. Scientific Opinion on the substantiation of health claims related to coffee, including chlorogenic acids from coffee, and protection of DNA, proteins and lipids from oxidative damage (ID 1099, 3152, 4301), maintenance of normal blood glucose concentrations (ID 1100, 1962), and contribution to the maintenance or achievement of a normal body weight (ID 2031, 4326) pursuant to Article 13(1) of Regulation (EC) No 1924/20061. 2011.

2 Farah A, Donangelo CM. Phenolic Compound in Coffee. Miniriview. Universidade Federal do Rio de Javeiro. 2006.

3 Farah, A, De Paulus T, Daniel P Morena DP, Trago LC, Martin PR. Cholorogenic Acid and Lactones in Regular and Water Decaffeinated Arabica Coffee. J.Agric. Food Chem. 54: 374-381. Brazil. 2006

4 Gilbert. S. F. Development Biology.2nd Ed. Sinaver Association, Inc. Publisher-Sunderland. Massahuset.Tesis. 1988.

5 Gratton JP, Morales-Ruiz M, Yasuko K, Avid F, Kenneth W, William CS. PI-3 Kinase and ATK to endothelial nitric oxide. J. Pharm and Exp. 299(30): 818-824. 2001

Monique CA, Theresa MH, Dennis F, Herbert MP, Epie B.. Induction of Vascular Endothelial Growth Factor Expressionand Hypoxia-inducible Factor 1? Protein by the OxidativeStressor Arsenite.Department of Medical Oncology, Vrije Universiteit Medical Centre, De Boelelaan 1117, Amsterdam 1081 HV,The Netherlands. 276 (51): 48066-48076. 2001

7 Nowak M, Madej JA, Dziegeil P. Intensity of Cox 2 Expression in Cell of Soft Tissue Fibrosarcomas in Dog As Related to Grade of Tumor Malignation. Bull Vet inst Pulawy. 51:275-279. 2007.

8 Watson ED, Cross JC.. Development of Structures and Transport Functions in the Mouse Placenta. J.Physiol. 20:180-193. 2005.

\author{
Received on 14.12.2017 Modified on 27.01.2018 \\ Accepted on 06.03.2018 $\quad$ C RJPT All right reserved \\ Research J. Pharm. and Tech 2018; 11(5):1830-1834
}

DOI: 10.5958/0974-360X.2018.00340.2 
